\title{
2006-1726: SEEING THE NANOSCALE: USING INTERACTIVES TO TEACH PROBE MICROSCOPY
}

\section{Olivia Castellini, University of Wisconsin-Madison}

Dr. Castellini was a postdoctoral researcher with the Interdisciplinary Education Group of the University of Wisconsin - Madison Materials Research Science and Engineering Center (UW MRSEC) and is now an exhibit developer at the Museum of Science and Industry.

GIna Walejko, University of Wisconsin-Madison

Ms. Walejko was an intern with the UW MRSEC Internships in Public Science Education Program.

\section{Carie Holladay, University of Wisconsin-Madison}

Ms. Holladay was an intern with the UW MRSEC Internships in Public Science Education Program.

Terra Theim, University of Wisconsin-Madison

Ms. Theim was an intern with the UW MRSEC Internships in Public Science Education Program.

\section{Anthony Cina, O'Keefe Middle School}

Mr. Cina is a teacher at O'Keefe Middle School and has participated in the UW MRSEC Research Experiences for Teachers Program.

\section{Greta Zenner, University of Wisconsin-Madison}

Ms. Zenner is Assistant Director of the Interdisciplinary Education Group of the UW MRSEC.

\section{Wendy Crone, University of Wisconsin-Madison}

Prof. Crone is an Associate Professor in the Department of Engineering Physics and Director of the Interdisciplinary Education Group of the UW MRSEC. 


\title{
Seeing the Nanoscale: Using Interactives to Teach Probe Microscopy
}

\begin{abstract}
Probe microscopes are key tools for surface characterization and nanoscale science. This category of instrumentation has enabled researchers both to investigate properties and to manipulate materials at the nanoscale. When introduced to nanoscale science and engineering, students frequently ask "How do we know it is there if we can't see it with our eyes?" To address this query, students of all ages can be introduced to the basic concepts of probe microscopy and given the opportunity to explore probe techniques. We have developed interactive exhibits and classroom activities that allow middle- and high-school students to detect and map topography, stiffness, and magnetic field. These products will be presented along with assessment data collected with the Rennie and McClafferty protocol for formative evaluation of interactive exhibits.
\end{abstract}

\section{Introduction}

Scanning probe microscopy (SPM) was first developed in the 1980s and uses an extremely fine probe tip to image a surface at the nanometer scale. Since its inception, this class of microscopy technique has proven invaluable for imaging, studying, and manipulating atoms and materials at the nanoscale. A number of different instruments fall into this category, including atomic force microscope (AFM), magnetic force microscope (MFM), and scanning tunneling microscope (STM). Researchers in nanoscale science and engineering (NSE) consider these SPM techniques to be an enabling factor that has allowed this emerging field to expand as rapidly as it has. Consequently, when considering an NSE education and outreach program, it is important to include materials that help students understand this fundamental tool.

The need for students to learn and appreciate the basic concepts behind NSE is increasing rapidly. Nanotechnology is inherently interdisciplinary in nature and is becoming increasingly important in fields such as medicine, electronics, and communications. It is estimated that by 2015, nanotechnology will be a trillion dollar industry employing five million workers ${ }^{1,2}$. However, there is concern that today's students will not be adequately prepared, thus creating a future bottleneck of skilled technicians and researchers and a citizenry ignorant of this new field. Primary and secondary educators need to instill knowledge fundamental to nanotechnology in their students now ${ }^{3}$ in order to prepare them for the boom in nanotechnology-related jobs that is predicted to occur and for the increased effect nanotechnology promises to have on society.

\section{Interactive Activities as a Vehicle for Teaching Nano}

Despite the increasing need, NSE currently does not occupy a place in the standard K-12 curriculum $^{4}$, and the main ideas important to this field are novel and difficult to grasp, especially for young students that are still learning about scale and powers of ten. Interactive activities, such as those found in museum exhibits and at outreach events, engage students and can help 
them understand challenging concepts in exciting ways ${ }^{5-7}$, which makes them ideal for communicating with students and the public about NSE and SPM.

Incorporating interactive activities on NSE into the K-12 science classroom is possible. At the University of Wisconsin-Madison Materials Research Science and Engineering Center (UW MRSEC), funded by the National Science Foundation (NSF), we have used both interactive exhibits and classroom activities to communicate two main ideas about SPM: that scientists use senses other than sight to image materials too small to be seen with the naked eye or traditional light microscopes, and that it is important to use tools that are on the same size scale as the sample to be studied. We present here interactive education materials developed as part of our NSF-funded Internships in Public Science Education (IPSE) and our Research Experiences for Teachers (RET) programs.

\section{Interactive Museum Exhibits}

As part of our IPSE program ${ }^{8}$, graduate student interns created museum exhibit prototypes targeted at middle- and high-school students. One of these exhibits uses interactives to help individuals understand how SPM works and how scientists measure and investigate structures at the nanoscale.

The SPM exhibit includes three interactive activities that allow visitors to use their hands as an analogy to the SPM probe. Students must rely on their sense of touch, rather than sight, to discern the hidden shapes. Two of the interactives are reach-in boxes that model different capabilities of atomic force microscopy (AFM), which runs an extremely fine probe tip over a sample surface. The tip can investigate a number of properties, but the two highlighted in the IPSE exhibit are stiffness and topography. The stiffness reach-in box has a shape made from two different kinds of foam, one stiffer than the other. Visitors press their hands on the hidden surface and discover the shape by feeling the arrangement of the varying stiffnesses of the two foams. Individuals who interact with the topography box feel a shape made out of bumps, analogous to an AFM tip scanning over a surface and sensing atomic-scale variations in the landscape. The third interactive models a magnetic force microscope, which uses a fine probe tip to measure the magnetic interactions between the tip and the sample surface. Visitors pass a magnetic wand over a surface to discover the magnetic shape hidden behind the opaque panel.

Formative evaluation of the SPM exhibit prototype was conducted using a three-stage method ${ }^{9}$ involving both passive observation and post-use interviews. Evaluation was conducted at two separate campus outreach events held at the University of Wisconsin-Madison (UW), during which hundreds of middle- and high-school students as well as families and general public audiences were observed interacting with the SPM exhibit prototype as well as an additional exhibit on liquid crystals ${ }^{10}$. The results of the visitor behavior observations revealed a strong difference in engagement among different age groups. For both exhibit prototypes, children and teen visitors were more likely to use the interactive features, while adults were more likely to simply attend to the text of the exhibit (Figure 1). Our results show that the interactive features of our museum exhibits especially interested younger visitors, suggesting that interactive activities and materials could capture students' attention in the classroom. Post-use interviews reveal that people understood that probe microscopes feel objects rather than imaging them 
optically, like traditional microscopes. Visitors particularly enjoyed discovering the shapes in the boxes, even requesting additional shapes to discern.

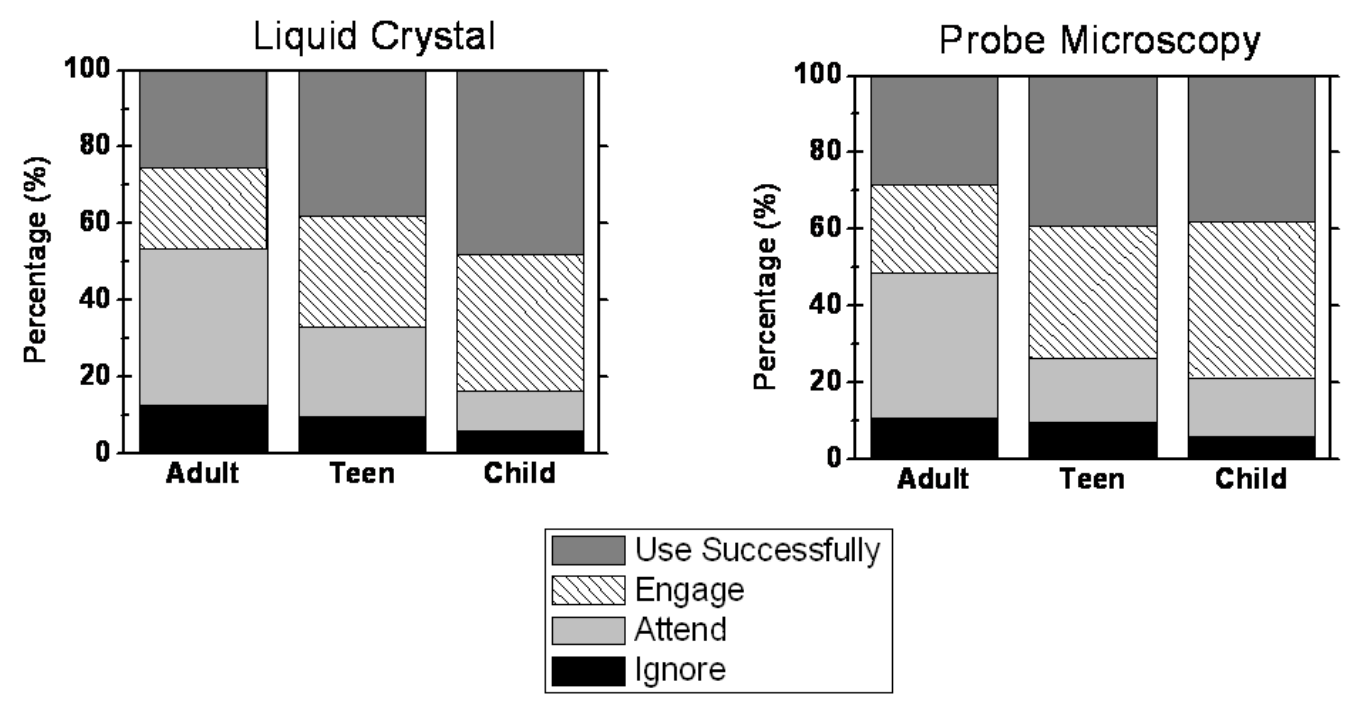

Figure 1. Visitor behavior observations. Level of engagement is defined as such:

- Use successfully: Visitor completes interactive feature of exhibit;

- Engage: Visitor touches, points toward or discusses exhibit with others;

- Attend: Visitor looks at or reads text on exhibit;

- Ignore: Visitor gives exhibit no more than cursory glance.

\section{Interactive Secondary Classroom Activity: "Seeing inside the Black Box"}

Field trips to a museum or outreach event are not always feasible, making in-classroom activities a necessity. The following activity, developed by a middle-school technology teacher as part of our NSF-funded Research Experiences for Teacher (RET) program, uses simple props to introduce middle-school students to SPM and the relationship between tool and sample size. A modified version of the full activity is available on our web-site at http://mrsec.wisc.edu/Edetc/modules/MiddleSchool/SPM/index.html, under "Mapping the Unknown."

In the activity, "Seeing inside the Black Box," students use a simple measurement tool - a wooden skewer - to image a mystery surface hidden inside a shoe box. Before class, the instructor prepares a landscape with a highly varied topography inside a sealed shoe box, using household objects such as cardboard, small balls, or wooden blocks. The instructor then pokes holes at regular, one-inch intervals in the box lid. During the activity, students measure the depth from the top of the box to the landscape surface by pushing the wooden skewer though the holes in the box top. The collected data points are used to make a 2-dimensional plot of the surface and then converted to a 3-dimensional model using mini-marshmallows (Figure 2). 

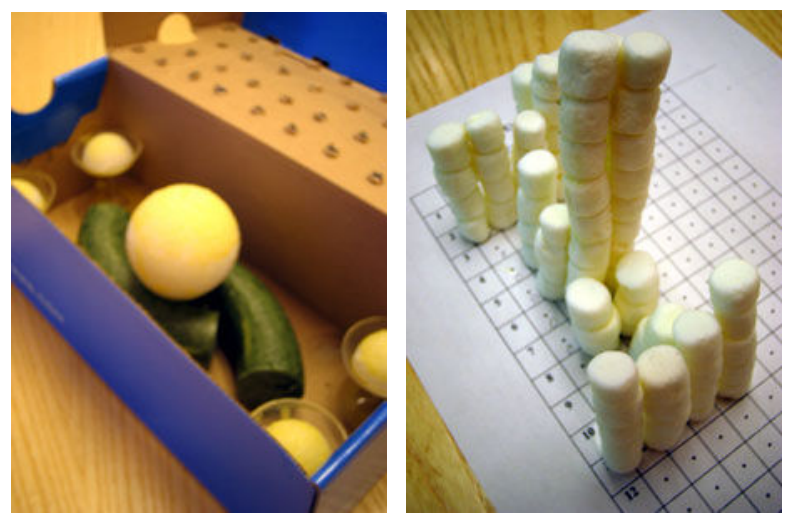

Figure 2. A mystery landscape inside a shoebox (left) and the corresponding marshmallow model (right).

The activity can be expanded to ensure that students understand that the level of detail they can "image" is directly related to the size of the probe. The precision of the data is also affecte $4 d$ by the sampling frequency, which is modeled by and the spacing of the holes in the box. If the distance between holes were zero, the students would be able to drag the probe freely across the surface and collect data from an infinite number of points. Any distance greater than zero gives students a more abstract representation of the mystery landscape. The time required for imaging can also be addressed. There is a correspondence between the level of detail in an image and the amount of time required to capture it - the finer the probe (i.e. the more holes in the box), the longer it would take to map the surface.

Students' learning can be assessed by creating different combinations of surfaces and probes and asking students to predict what would happen if they tried to map the surfaces with the probes. The assessment can be performed as a lab-practical-style exam, with the surfaces and probes set up at different stations around the classroom, or as a paper-and-pencil test using pictures of the surfaces and probes, as in Figure 3.

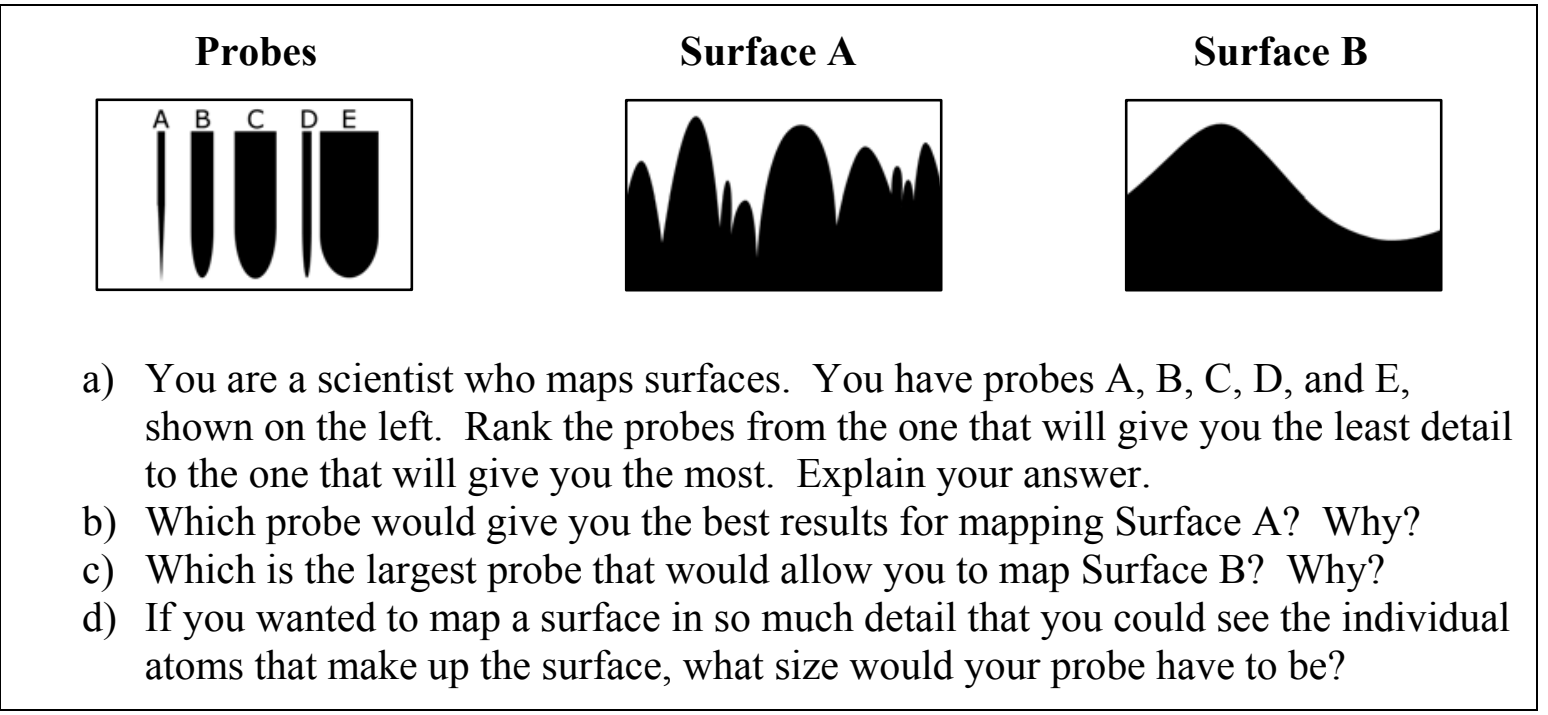

Figure 3. Sample assessment question for the "Seeing inside the Black Box" activity. 


\section{Conclusion}

The exhibit interactives and the classroom activity both address science standards in modeling and inquiry important for the middle-school level. Through their own use of probes - whether it be their hand, a wand, or a skewer - students discover that they can learn about a surface and its properties by indirect means and visualize its shape from the data they collect. These are critical scientific skills that students are able to practice in the context of nanoscale science, thus helping to educate future consumers, voters, technicians, and scientists about this emerging field in an innovative manner.

\section{Acknowledgements}

The products discussed were developed under the following National Science Foundation grants at the University of Wisconsin - Madison: Materials Research Science and Engineering Center (MRSEC) on Nanostructured Interfaces (\#DMR-0079983 and \#DMR-0520527) and Internships in Public Science Education (IPSE): Making the Nanoworld Comprehensible (\#DMR-0424350).

\section{References}

1. $\quad$ Roco, M. C. Journal of Nanoparticle Research 2003, 5, 181-189.

2. Roco, M. C. Nature Biotechnology 2003, 21, (10), 1247-1249.

3. Smaglik, P. Naturejobs 2002, 418, 4-6.

4. National Research Council (NRC). 1996. National Science Education Standards.

Washington D.C.: National Academy Press. Also available on-line: Retrieved December 21, 2005, from http://www.nap.edu/readingroom/books/nses/html/

5. $\quad$ Csikszentmihalyi, M.; Hermanson, K. Museum News 1995, 74, (3), 34-38.

6. $\quad$ Rennie, L. J.; McClafferty, T. P. Studies in Science Education 1996, 27, 5398.

7. Sandifer, C. Journal of Research in Science Teaching 2003, 40, (2), 121-137.

8. Internships in Public Science Education, University of Wisconsin-Madison. Madison:

University of Wisconsin-Madison, Board of Regents, 2005 [cited 21 December 2005]. Available from World Wide Web: http://mrsec.wisc.edu/Edetc/IPSE_exhibits/index.html.

9. Rennie, L.; McClafferty, T. Handbook for Formative Evaluation of Interactive Exhibits; Science and Mathematics Education Centre Curtin University of Technology: Perth, Western Australia, 1996.

10. Castellini, O. M.; Holladay, C. E.; Walejko, G. K.; Theim, T. J.; Zenner, G. M.; Krajniak, P.; Crone, W. C. to appear in Journal of Materials Education. 